\title{
A comprehensive study of artificial intelligence applications for soil temperature prediction
}

\author{
Hanifeh Imanian ${ }^{1, *}$, Juan Hiedra Cobo ${ }^{2}$, Pierre Payeur ${ }^{3}$, Hamidreza Shirkhani ${ }^{2}$ and Abdolmajid Mohammadian ${ }^{1}$ \\ 1 Civil Engineering Department, University of Ottawa, Ottawa, Canada; himania3@uottawa.ca (H.I.); \\ majid.mohammadian@uottawa.ca (A.M.) \\ 2 National Research Council Canada, Ottawa, Canada; juan.hiedracobo@nrc-cnrc.gc.ca (J.C.); ha- \\ midreza.shirkhani@nrc-cnrc.gc.ca (H.S.) \\ 3 Computer Science Engineering Department, University of Ottawa, Ottawa, Canada; ppayeur@uottawa.ca \\ * Correspondence: himania3@uottawa.ca
}

\begin{abstract}
Soil temperature is a fundamental parameter in water resources and irrigation engineering. A cost-effective model which can forecast soil temperature accurately is extensively needed. Recently, many studies have applied artificial intelligence (AI) at both surface and underground levels for soil temperature prediction. In the present study, attempts are made to deliver a comprehensive and detailed assessment of the performance of a wide range of AI approaches in soil temperature prediction. In this regard, thirteen approaches, from classic regressions to well-established methods of random forest and gradient boosting to more advanced AI techniques like multi-layer perceptron and deep learning, are taken into account. Meanwhile, great varieties of land and atmospheric variables are applied as model input. A sensitivity analysis was done on input climate variables to determine the importance of each variable in predicting soil temperature. This examination reduced the number of input variables from 8 to 7 , which decreased the simulation load. Also, this showed air temperature and solar radiation plays the most important roles in soil temperature prediction, while precipitation can be neglected in forecast AI models. Comparison of soil temperature predicted by different AI models showed that deep learning demonstrated the best performance with R-squared of 0.980 and NRMSE of $2.237 \%$, followed by multi-layer perceptron with R-squared of 0.980 and NRMSE of $2.266 \%$ and stacking method with R-squared of 0.981 and NRMSE of $2.217 \%$.
\end{abstract}

Keywords: artificial intelligence; climate forecast; deep learning; ensemble model; multi-layer perceptron; neural network; regression; soil temperature; stacking method

\section{Introduction}

Soil temperature is a pivotal parameter in geo-environmental and geotechnical engineering. Soil temperature prediction is significant for atmospheric models, numerical hydrological and land surface hydrological processes, as well as land-atmosphere interactions [1,2]. In addition, in some other fields such as water resources and hydrologic engineering, soil temperature is an important factor [3]. The soil temperature is a catalyst for many biological processes. It influences soil moisture content, aeration and availability of plant nutrients, which are necessary for plant growth. So, it is essential to measure or estimate this parameter with a reasonable precision. Therefore, an accurate and cost-effective model which can predict soil temperature accurately is extensively needed [4-6].

There are two common ways of obtaining soil temperature: direct measurement and indirect prediction using numerical models [7]. Since soil temperature is a stochastic parameter like other climatic parameters, researchers use the following approaches to calculate it: statistical models and machine learning methods [8]. Statistical models use historical time series to estimate soil temperature in the future.

The commonly used method for time series forecasting is stochastic modeling such as auto-regressive moving average (ARMA) and the auto-regressive integrated moving average (ARIMA) [9]. The statistical methods assume that changes in the statistical 
properties of soil temperature data series in the future would be similar to those in the past. This means that large amounts of data are required for long-term prediction.

Bonakdari et al. (2019) and Zeynoddin et al. (2020) proposed a linear stochastic method to model daily soil temperature with sufficient knowledge of time series structure $[6,9]$. Recently, the use of artificial intelligence (AI)-based techniques for predicting realworld problems has grown enormously. Many studies have applied AI models at both surface and under-ground levels for soil temperature prediction. George (2001) made use of a multi-layer neural network for the weekly mean soil temperature prediction during 1 year [10].

Monthly soil temperature was modeled using a 3-layer artificial neural network (ANN) constructed by Bilgili (2010) [11]. He used meteorological variables of atmospheric temperature, atmospheric pressure, relative humidity, wind speed, rainfall, global solar radiation and sunshine duration to make predictions at five depths below the ground level and compared them with linear and nonlinear regression results.

Ozturk et al. (2011) developed feed-forward artificial neural network models to estimate monthly mean soil temperature at five depths from 5 to $100 \mathrm{~cm}$ under the ground using meteorological data such as solar radiation, monthly sunshine duration and monthly mean air temperature [12].

Zare Abyaneh et al. (2016) used ANNs and co-active neuro-fuzzy inference system (CANFIS) for the estimation of daily soil temperatures at six depths from 5 to $100 \mathrm{~cm}$ underground using only mean air temperature data from a 14-year period as input data [13].

Adaptive neuro-fuzzy inference system (ANFIS), multiple linear regression (MLR) and ANN models were developed by Citakoglu (2017) to predict soil temperature data in monthly units at five depths from 5 to $100 \mathrm{~cm}$ below the soil surface using monthly air temperatures and monthly precipitation for at least 20 years [14].

Himika et al. (2018) made use of various existing regression and machine learning models to propose an ensemble approach to predict land temperature [15]. The chosen models were decision tree, variable ridge regression and conditional inference tree.

Delbari et al. (2019) evaluated the performance of a support vector regression (SVR)based model in estimating daily soil temperature at 10,30 and $100 \mathrm{~cm}$ depth at different climate conditions [16]. Climatic data used as inputs for the models were air temperature, solar radiation, relative humidity, dew point, and the atmospheric pressure. They compared the obtained results with classical MLR and found that SVR performed better in estimating soil temperature at deeper layers.

A study by Alizamir et al. (2020) compared four machine learning techniques, extreme learning machine (ELM), artificial neural networks (ANN), classification and regression trees and group method of data handling in estimating monthly soil temperatures [3]. They used monthly climatic data of air temperature, relative humidity, solar radiation, and windspeed at four different depths of 5 to $100 \mathrm{~cm}$ as model inputs. ELM was found to generally perform better than the others in estimating monthly soil temperatures.

Li et al. (2020) presented a novel scheme for forecasting the hourly soil temperature at five different soil depths [17]. They developed an integrated deep bidirectional long short-term memory network (BiLSTM) and fed their model with air temperature, wind speed, solar radiation, relative humidity, vapor pressure and dew point. Six benchmark algorithms were chosen to prove the relative advantages of the proposed method, namely, three deep learning methods, i.e., LSTM, BiLSTM and deep neural network (DNN), and three traditional machine learning methods: random forest (RF), SVR, and linear regression.

The proposed model of Penghui et al. (2020) is a hybridization of adaptive neurofuzzy inference system with optimization methods using mutation salp swarm algorithm and grasshopper optimization algorithm (ANFIS-mSG) [18]. The prediction of daily soil temperatures was conducted based on maximum, mean and minimum air temperature. The results were compared with seven models, including classical ANFIS, hybridized ANFIS model with grasshopper optimization algorithm (GOA), salp swarm algorithm 
(SSA), grey wolf optimizer (GWO), particle swarm optimization (PSO), genetic algorithm (GA), and Dragonfly Algorithm (DA).

Shamshirband et al. (2020) modeled air temperature, relative humidity, sunshine hours and wind speed using multilayer perceptron (MLP) algorithm and SVM in hybrid form with the firefly optimization algorithm (FFA) to estimate soil temperature at 5, 10 and $20 \mathrm{~cm}$ depth [19].

In a study by Seifi et al. (2021), hourly soil temperatures at 5, 10, and $30 \mathrm{~cm}$ depth were predicted applying ANFIS, SVM, MLP and radial basis function neural network with optimization algorithms of SSA, PSO, FFA and sunflower optimization (SFO) [4]. They used air temperature, relative humidity, wind speed and solar radiation as input information and found that wind speed did not have high coherence with soil temperature. Generalized likelihood uncertainty estimation approach was implemented to quantify model uncertainty and concluded that ANFIS-SFO produced the most accurate performance.

Hao et al. (2021) proposed a model called convolutional neural network based on ensemble empirical mode decomposition (EEMD-CNN) to predict soil temperatures at three depths of 5 to $30 \mathrm{~cm}$ [1]. They used Statistical properties of the maximum, mean, minimum and variance air temperature as the meteorological input information. The results were compared with four models: persistence forecast (PF), backpropagation neural network, LSTM and EEMD-LSTM.

In a similar study, a convolutional 3D deep learning model with ensemble empirical mode decomposition (EEMD) was proposed by Yu et al. (2021) to predict soil temperatures over 1, 3 and 5 days at a depth of $7 \mathrm{~cm}$ underground [2].

The literature review shows that there are some gaps in the knowledge of AI application in prediction of soil temperature. First, there is an absence of a comprehensive and detailed assessment of the performance of different artificial intelligence approaches, from linear regression to complicated advanced techniques in soil temperature estimation. Second, in the context of atmospheric variables used as input data for AI models, previous studies have usually used limited atmospheric variables, while in the current investigation, a wide range of variables have been used. Although several researchers have developed codes equipped with some AI models, they have focused on limited meteorological parameters, mostly air temperature. There are many other climate data that affect soil temperature, directly or indirectly. Therefore, the impact of other land and atmospheric variables needs to be further studied.

The main purpose of this study is to evaluate the performance of a wide range of AI approaches on soil temperature prediction using various land and atmospheric variables. In this article, 13 methods, from classic regressions to well-established methods of random forest and gradient boosting to advanced AI techniques like ANFIS, ANN and deep learning are taken into account. Meanwhile, a broad selection of variables from a comprehensive reanalysis of ERA5 datasets have been chosen as input parameters for the developed prediction model to consider different aspects of the problem.

The rest of the paper is organized as follows: Section 2 describes the study area and involved parameters, introduces the applied AI approaches and reviews the methodology of study. The evaluation metrics are also presented in this section. The subsequent section defines datasets and input information, presents the results and compares them with the actual data. Discussion on the performance of the different AI methods using error metrics and confidence bands and sensitivity analysis of outcome to input parameters are given in section 4 . The last section presents the concluding remarks and future study suggestion.

\section{Materials and Methods}

\subsection{Study area and dataset}

The climate data used in the present study are obtained from ERA5. It was downloaded from freely accessible website of Climate Data (https://cds.climate.copernicus.eu/, accessed on 1 July 2021). ERA5 is the fifth-generation atmospheric reanalysis of the global 
climate covering the period from 1950 to present. It provides hourly estimates of a large number of atmospheric, land and oceanic climate variables in a gridded-base format with a regular latitude-longitude grid. The data coverage is global with horizontal resolution of $0.25^{\circ} \times 0.25^{\circ}$ and resolves the atmosphere using 137 levels from the surface up to a height of $80 \mathrm{~km}$. ERA5 includes information about uncertainties for all variables at reduced spatial and temporal resolutions. ERA5 combines vast amounts of historical observations into global estimates using advanced modelling and data assimilation systems.

The study area is Ottawa, the capital city of Canada $\left(45.4^{\circ} \mathrm{N}, 75.7^{\circ} \mathrm{W}\right)$, located in the southeast of the country, in the province of Ontario. Figure 1-(a) shows the geographical location of the considered site used in this study [20]. Ottawa has a semi-continental climate with four distinct seasons. It has a warm, humid summer and a very cold and harsh winter.

Six stations represent the city of Ottawa, which are specified with red circles in Figure $1-(b)$. The stations cover an area of approximately $30 \times 40 \mathrm{~km}$. The coordinates of the stations are mentioned in Table 1.

Table 1. Coordinates of considered stations in city of Ottawa.

\begin{tabular}{cccccc}
\hline Station no. & latitude & longitude & Station no. & latitude & longitude \\
\hline$\# 1$ & $45.25^{\circ} \mathrm{N}$ & $75.50^{\circ} \mathrm{W}$ & $\# 4$ & $45.50^{\circ} \mathrm{N}$ & $75.50^{\circ} \mathrm{W}$ \\
$\# 2$ & $45.25^{\circ} \mathrm{N}$ & $75.75^{\circ} \mathrm{W}$ & $\# 5$ & $45.50^{\circ} \mathrm{N}$ & $75.75^{\circ} \mathrm{W}$ \\
$\# 3$ & $45.25^{\circ} \mathrm{N}$ & $76.00^{\circ} \mathrm{W}$ & $\# 6$ & $45.50^{\circ} \mathrm{N}$ & $76.00^{\circ} \mathrm{W}$ \\
\hline
\end{tabular}

The used variables were the hourly weather conditions including air temperature at $2 \mathrm{~m}$ above the surface (Kelvin), total precipitation $(\mathrm{m})$, surface pressure (Pa), evaporation ( $\mathrm{m}$ of water), instantaneous wind gust at $10 \mathrm{~m}$ above the surface $(\mathrm{m} / \mathrm{s})$, dewpoint temperature $2 \mathrm{~m}$ above the surface (Kelvin), surface net solar radiation (J/m2) and surface net thermal radiation $(\mathrm{J} / \mathrm{m} 2)$. The valid data was from 1st June 2020 to 31st August 2020, a total of 92 days [21]. The information of these variables in the six mentioned stations are considered as model inputs. Then, approximately 106,000 pieces of climatic information was gathered as the AI model's input. The output of each AI model predicted hourly soil temperature in Kelvin at the layer between 0 to $7 \mathrm{~cm}$ underground.



(a) 


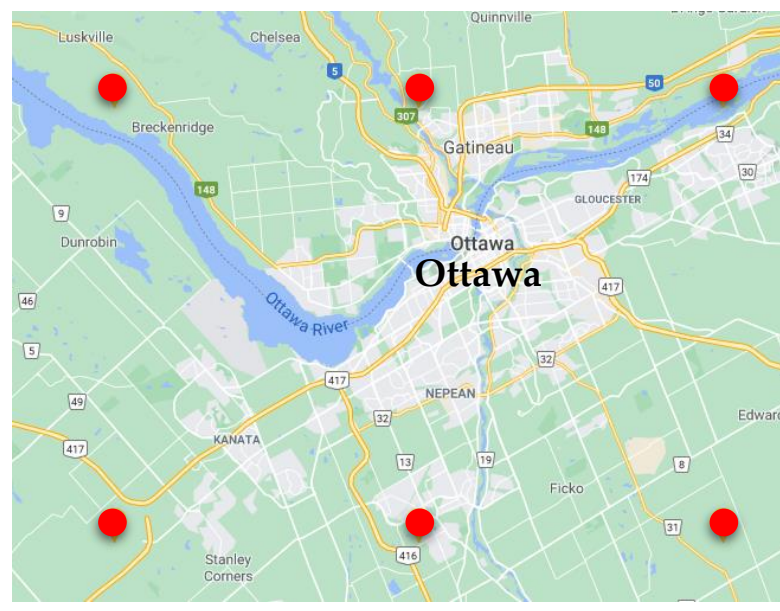

(b)

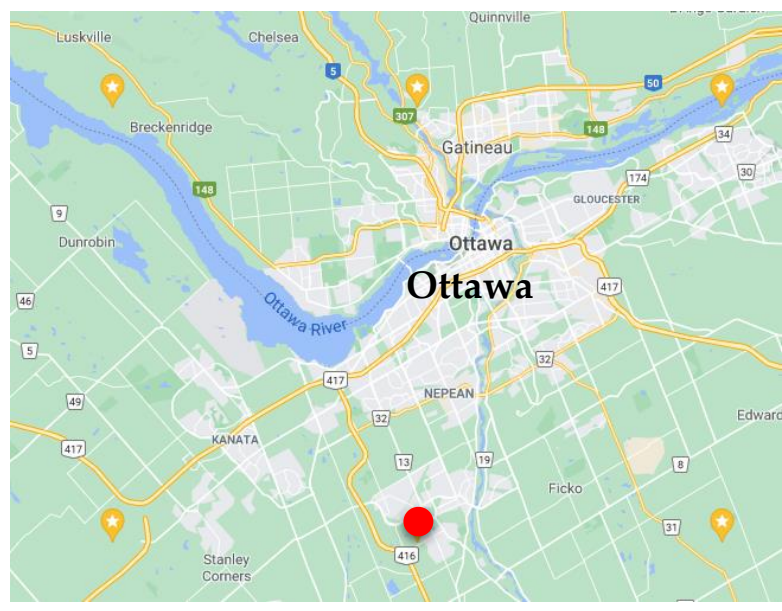

(c)

Figure 1. Location of the a) The site (Ottawa) considered in the present study, b) six stations representing Ottawa, c) one selected station.

\subsection{Descriptions of artificial intelligence algorithms}

A wide range of AI approaches are applied in the developed numerical model as described below.

\subsubsection{Linear regression, Ridge, Lasso and Elastic Net}

Four different linear models were applied in the developed code including: Linear regression, Ridge, Lasso and Elastic Net. Linear regression is the most basic form of a linear model for minimizing the residual sum of squares. So, the objective function is as follows $[11,14,17,22]$ :

$$
\sum(y-X w)^{2}
$$

where $y$ is the actual value, $w$ is the input value and $w$ is weight.

Ridge is a linear model that imposes a penalty on the sum of squared value of the weights, resulting in a group of weights that are more evenly distributed. The objective function called L2 regularization:

$$
\sum(y-X w)^{2}+\alpha \sum w^{2}
$$

where $\alpha$ is a non-negative hyperparameter that controls the magnitude of the penalty. In the presents study, $\alpha$ was set to 0.1 by iteration for Ridge model.

For Lasso, a modification of linear regression is applied, in which the model is penalized for the sum of absolute values of the weights called L1 regularization:

$$
\frac{1}{2 m} \sum(y-X w)^{2}+\alpha \sum|w|
$$

In the presents study, $\alpha$ was set to 0.001 by iteration for Lasso model.

Elastic Net is a combination of the two last models such that both regularizations related to Ridge and Lasso models are exerted on the linear regression. Parameter $\rho$ defines the ratio of penalties. If $\rho$ is zero, the penalty would be L2 regularization; if $\rho$ is one, the penalty would be L1 regularization.

$$
\frac{1}{2 m} \sum(y-X w)^{2}+\alpha * \rho \sum|w|+\frac{\alpha}{2}(1-\rho) * \sum w^{2}
$$

In the presents study, $\rho$ was set to 0.5 so it was a combination of Ridge and Lasso models. Also, $\alpha$ was set to 0.0001 , obtained by iteration for Elastic Net model.

\subsubsection{Nearest Neighbors method}

In the Nearest Neighbors method, learning is based on the fixed number of nearest neighbors of each query point, or on the neighbors within a fixed radius of the query point. It can be weighted uniformly or proportional to distance. 
The present study implemented learning on k nearest neighbors' points. Parameter $\mathrm{k}$ was set to 3 after calibration. Also, a uniform weight function was used in prediction, which means each point in the local neighborhood contributes uniformly to the classification of a query point.

\subsubsection{Decision Tree, Random Forest, Gradient Boosting and Extreme Gradient Boosting}

There is another learning method called Decision Trees. This method's goal is to create a model that predicts the value of a target variable by learning simple decision rules inferred from the data features. In other words, a tree can be seen as a piecewise constant approximation.

Ensemble learning method is a technique that combines predictions from multiple machine learning algorithms in order to make a more accurate prediction than a single model. Some ensemble models are developed based on decision tree like Random Forest and Gradient Boosting.

Random Forest is a meta estimator that fits a number of decision trees on various subsets of the dataset. A RF operates by constructing several decision trees during training time and outputting the mean of the classes as the prediction of all the trees. Several trees run in parallel with no interaction amongst them in this method [7]. In the presents study, the number of decision trees in the random forest model was set to 1000 after sensitivity analysis.

Gradient Boosting fits a decision tree, repeatedly on the differentiable loss functions. This method builds one Decision Tree at a time, where each new tree helps to correct errors made by the previously trained tree. Gradient boosting is fairly robust against overfitting so a large number of boosting stages usually results in better performance. In the present study, the number of boosting stages to perform was set to 1000 after sensitivity analysis. More reparation did not improve the results significantly.

Extreme Gradient Boosting (XG Boost) builds a model by a set of trees, reduces the errors, and builds a new model in subsequent iterations. Unlike Gradient Boosting method, XG Boost implements some regularization; therefore, it helps to reduce overfitting. Also, it is much faster compared to Gradient Boosting.

There are some hyperparameters that should be considered tuning for the XG Boost model: number of used decision trees that would often be better if have more, tree depth that controls how specialized each tree is to the training dataset and learning rate that controls the amount of contribution of each decision tree model on the ensemble prediction. In the present study, the number of decision trees, tree depth and learning rate were set to 100,6 and 0.3 , respectively.

\subsubsection{Support Vector Machine}

The basic idea of Support Vector Machines is to map the original data into a feature space with high dimensionality through a non-linear mapping function and construct an optimal hyperplane in the new space. In case of regression, a hyperplane is to be constructed that lies close to as many points as possible. It means the optimal hyperplane it seeks is not to maximize the separation distance between two or more kinds of sample points like SVM, but to minimize the total deviation between sample points and hyperplane $[23,24]$.

When solving nonlinear problems, SVR applies kernel function to map the nonlinear regression problem to the space of higher latitude, so that it can find an optimal hyperplane to separate the sample points. The radial basis function kernel is widely used for SVM models and was applied in the current study:

$$
K\left(X_{i}, X_{j}\right)=\exp \left(-\gamma \sum\left(X_{i}-X_{j}\right)^{2}\right)
$$

where $X_{i}, X_{j}$ are two point and the hyperparameter $\gamma$ passes the reciprocal of number of features, which was set to 8 in the present study.

\subsubsection{Stacking method}

Another group of methods is called stacking or Stacked Generalization. Stacking is an ensemble machine learning algorithm that learns how to best combine the predictions 
from multiple well-performing machine learning models. In this paradigm, the outputs of some aforementioned individual estimators are gathered and an additional regressor is used to compute the final prediction. Stacking often ends up with a model which is better than any individual intermediate model [25].

In the present study, different combinations of estimators are tried and eventually three methods, Random Forest, SVM and Ridge, computed the best outcome.

In the first step, the stacking method uses some predictors, which consist of a list of machine learning methods stacked together in parallel on the input data. Herein, Random Forest and SVM methods were applied as predictors. At the second step of stacking method, a final predictor is employed that uses the predictions of the first estimators as input. The final predictor is a machine learning regressor which was chosen to be the Ridge method in the current study.

\subsubsection{Multi-Layer Perceptron}

Multi-Layer Perceptron (MLP), a class of feedforward ANN, is a non-linear function approximator in layers using back propagation with no activation function in the output layer. It used rectified linear unit function as the activation function in the hidden layers [7,8,26-29]:

MLP uses different loss functions depending on the problem type. For case of prediction, MLP uses the square error loss function; written as:

$$
\frac{1}{2} \sum(y-X w)^{2}+\frac{\alpha}{2} \sum w^{2}
$$

Starting from initial random weights, MLP minimizes the loss function by repeatedly updating these weights. After computing the loss, a backward pass propagates it from the output layer to the previous layers, providing each weight parameter with an update value meant to decrease the loss. The algorithm stops when it reaches a pre-set maximum number of iterations, or when the improvement in loss is below a certain, small number.

In the current study 700 hidden layers were used in the MLP model after sensitivity analysis. The activation function for the hidden layers was rectifier linear unit (Relu), which passes the maximum of the variable and zero. The solver for weight optimization was Adam which is a stochastic gradient-based optimizer. Maximum number of iterations was 5000 , which determines the number of epochs means how many times each data point will be used.

\subsubsection{Deep Learning}

Deep Learning is a subfield of ANN consisting of large neural networks with significant amounts of data. The word "deep" is referring to the depth of layers in a neural network. A neural network that consists of more than three layers, which would be inclusive of the inputs and the output, can be considered a deep learning algorithm $[17,27]$.

In deep learning method, the number of epochs is the number of times that the entire training dataset is shown to the network during training. In the current study, this hyperparameter was set to 100. The optimization algorithm used to train the network was Adam. The activation function controls the non-linearity of individual neurons was Relu. The number of neurons in the hidden layer that controls the representational capacity of the network was set to 30 after tuning.

\subsubsection{Adaptive Neuro-Fuzzy Inference System}

Adaptive Neuro-Fuzzy Inference System (ANFIS) models combine fuzzy systems and the learning ability of neural networks. ANFIS is considered an ANN model doing the preprocessing step by converting numeric values into fuzzy values [5,26,30-32].

The toolbox feature of the ANFIS forms a fuzzy inference system whose membership structure or parameters can be calibrated either by using a backpropagation method alone or combining with the least-squares-type method. To create an inference system, five different layers, namely fuzzy layer, product layer, normalized layer, de-fuzzy layer, and the total output layer, are used. Each consists of different adaptive nodes that exert changeable and fixed factors on input values. 
The ANFIS rules are presented in the following form: $y=p x_{1}+q x_{2}+r$

Where $x_{1}, x_{2}$ are input variables from corresponding fuzzy sets, $y$ is the output, and $p, q, r$ are constant parameters.

\subsection{Methodological overview}

The collected data has been split into two parts randomly. The first part, including $65 \%$ of the data, is used for the training phase, while the remaining $35 \%$ of the data is used as the testing set.

In the splitting part of the AI models, the dataset was divided to train and test subsets randomly. It means that data was shuffled first, then $65 \%$ of shuffled data was used for training phase and the remaining data was set aside as testing data. The considered AI approach was fitted to the train subset and the accuracy of training step was calculated based on several error measures as described in equations 9 to 14 . In the next step, the fitted AI model was applied to the test subset for prediction purpose. Again, the accuracy of testing step was calculated.

Different explanations can be made on the applied AI models considering the accuracy of training and testing steps. If the model performs much better on the training set than on the test set, then we are likely overfitting. Underfitting refers to a model that can neither model the training data nor the testing data. The sweet spot between underfitting and overfitting, which shows the good performance of a machine learning algorithm on both training and testing data, is a good fit $[5,27]$.

The accuracy results of the training and testing stages of each applied AI model are presented in the Table 2 . The values show good performance of all models on both training and testing data, which proves the dataset is not prone to overfitting.

Moreover, to ensure that splitting does not affect the model performance, cross-validation (CV) metrics are employed in the developed codes. In this function, splitting the data was done $\mathrm{k}$ consecutive times (here typical times of 5 were chosen) with different splits each time. Fitting the model and computing the scores were done for each iteration. The mean and standard deviation of scores are presented in the Table 2 for every AI model. The fact that for all models, the mean is near 1.0 and the standard deviation is negligible, shows that random splitting was successful in the cross-validation evaluation and did not lead to overfitting in the considered case.

Table 2. Checking overfitting and cross validation

\begin{tabular}{|c|c|c|c|c|c|c|c|c|c|c|c|c|c|}
\hline 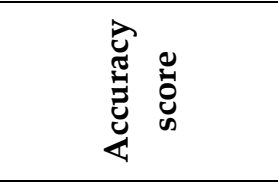 &  & $\begin{array}{l}0 \\
\text { के } \\
\text { త }\end{array}$ & 萬 &  & 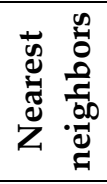 & $\stackrel{L}{\simeq}$ &  & $\begin{array}{l}\vec{\omega} \\
0 \\
0 \\
0 \\
0 \\
x\end{array}$ & $\sum_{\infty}$ & 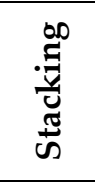 & $\stackrel{B}{z}$ & ڤัٌ & $\frac{n}{Z}$ \\
\hline training $\mathrm{R}^{2}$ & 0.96 & 0.96 & 0.96 & 0.96 & 0.96 & 0.99 & 0.99 & 0.99 & 0.99 & 0.98 & 0.98 & 0.98 & 0.96 \\
\hline testing $\mathrm{R}^{2}$ & 0.94 & 0.94 & 0.94 & 0.94 & 0.91 & 0.94 & 0.95 & 0.94 & 0.97 & 0.96 & 0.96 & 0.97 & 094 \\
\hline Mean K-fold CV & 0.95 & 0.95 & 0.95 & 0.95 & 0.89 & 0.95 & 0.96 & 0.95 & 0.97 & 0.96 & 0.96 & 0.96 & 0.95 \\
\hline Sdev K-fold CV & 0.01 & 0.01 & 0.01 & 0.01 & 0.03 & 0.02 & 0.01 & 0.02 & 0.01 & 0.01 & 0.01 & 0.01 & 0.01 \\
\hline
\end{tabular}

The air temperature, precipitation, surface pressure, evaporation, instantaneous wind speed, dewpoint temperature, solar radiation and thermal radiation are the atmospheric variables used as the inputs of the benchmark algorithm, and the soil temperature at a depth of $0-7 \mathrm{~cm}$ underground is the output of the model.

With the aim of making model training less sensitive to the scale of parameters and allowing our models to converge to better weights and, in turn, lead to a more accurate model, the data has been normalized in a way that removes the mean, and scales each variable to unit variance. Scaling happened independently on each parameter by computing the relevant statistics on the samples in the training set. Mean and standard deviation 
were then stored to be used on later data using denormalization. The same procedure was employed for testing data before prediction.

The overall flow of the simulation is illustrated in Figure 2.

The applied programming language in this study was Python version 3.9. Python is a high-level, object-oriented general-purpose, interactive language which is widely used for data analysis and machine learning. Also, Spyder version 5.1.5 was employed which is a scientific Python development environment. The processor of used device was 11th Gen Intel Core 15 @ 2.40GHz and installed RAM was 8.00 GB.

To facilitate the assessment of the model performance, the outputs of applied AI models need to be compared. Several error indicators are employed to measure the quality of modeling including maximum residual error (MaxE), mean absolute error (MAE), mean square error (MSE), root mean square error (RMSE), normalized root mean square error (NRMSE) and coefficient of determination (R-squared). The evaluation metrics are defined as:

$$
\begin{array}{ll}
M a x E=\operatorname{Max}\left(y_{o b s}-y_{\text {calc }}\right) & \text { optimal value: } 0 \\
M A E=\frac{\sum\left|y_{o b s}-y_{c a l c}\right|}{n} & \text { optimal value: } 0 \\
M S E=\frac{\sum\left(y_{o b s}-y_{c a l c}\right)^{2}}{n} & \text { optimal value: } 0 \\
R M S E=\sqrt{\frac{\sum\left(y_{o b s}-y_{\text {calc }}\right)^{2}}{n}} & \text { optimal value: } 0 \\
N R M S E=\frac{R M S E}{\left[M a x\left(y_{o b s}\right)-M i n\left(v_{o b s}\right)\right]} & \text { optimal value: } 0 \\
R^{2}=1-\frac{\sum\left(y_{o b s}-y_{c a l c}\right)^{2}}{\sum\left(y_{o b s}-\bar{y}_{c a l c}\right)^{2}} & \text { optimal value: } 1
\end{array}
$$

where $y_{\text {obs }}$ is the observed value, $y_{\text {calc }}$ is the predicted value by the AI mode, $\overline{y_{\text {calc }}}$ is the mean of calculated values and $n$ is the number of data.

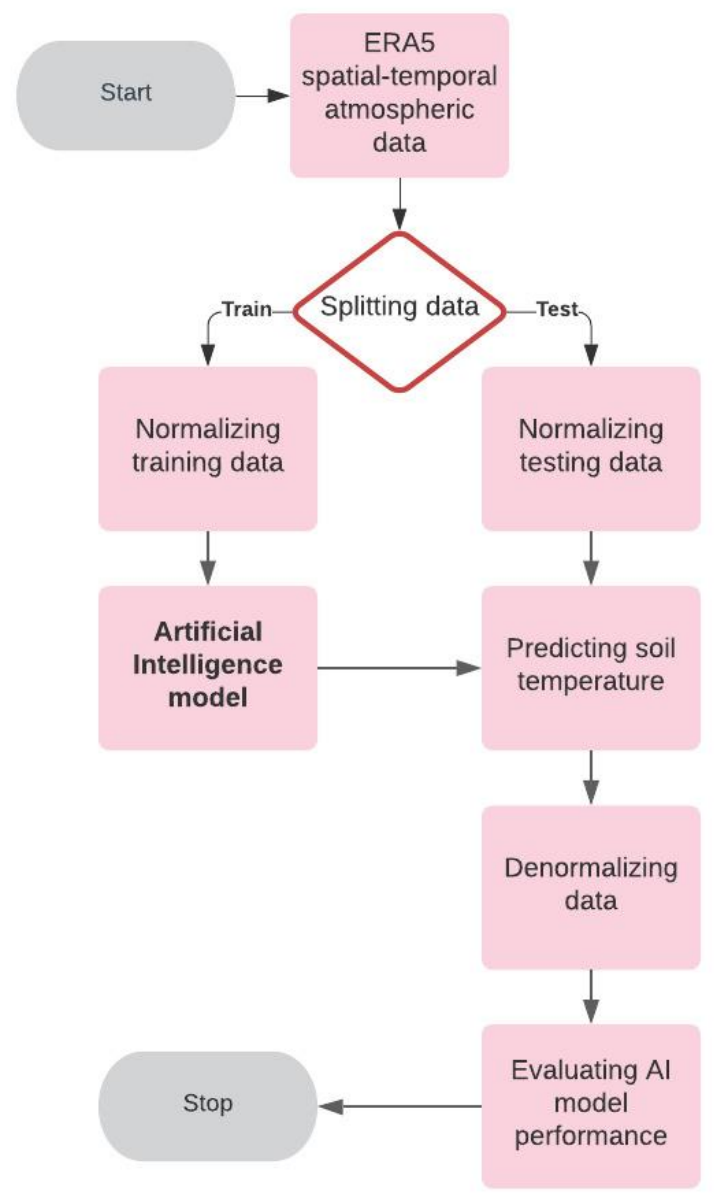


Figure 2. Algorithm of the developed numerical model in the present study.

\section{Results}

Before applying the models to all stations representing Ottawa, one station (station \#2 in Table 1) was selected and AI models were applied. The location of the station was at the mid-south of Ottawa, with coordinates of $45.25^{\circ} \mathrm{N}$ and $75.75^{\circ} \mathrm{W}$, which is demonstrated in Figure 1-(c). As mentioned earlier, eight hourly climate parameters were used as input variables. The data was gathered from 1st to 31st of July 2020, a total of 31 days that leads to approximately 6,000 pieces of climatic information.

According to what was stated, AI models were applied for two sets of data; first, on a confined database with a limited quantity of information based on station \#2 mentioned in Figure 1-(c) and is called limited dataset in the current work. Second, an extensive collection of data based on the information of six stations mentioned in Figure 1-(b) and is called big dataset in the present work.

After applying each developed AI model on these two datasets, evaluation of the model's performance was carried out separately; then, a comprehensive assessment was finally performed.

The primary step of modeling was splitting the data into two groups. Although the data was split for training and testing purposes randomly, the training and testing data was maintained for all models. So, all AI models were trained and tested with the same set of data. After the model training procedure, the model was fed with testing data as input, and prediction results were obtained. The predicted outcomes and real values were simultaneously reshaped into a 1-dimensional series, and the performance of the models was evaluated using the error metrics.

The developed model was executed each time, employing one of the 13 abovementioned AI techniques, once on the limited database and once on the big dataset. Hence, 26 sets of predicted data were obtained.

The residuals of soil temperature, the difference between the actual and the predicted values obtained from each AI model, were calculated and illustrated in Figure 3 as a box plot.

Box plot is a graphical method for demonstrating the locality, spread and skewness groups of numerical data. This diagram is a standardized way of displaying the five significant summary numbers of minimum $\left(Q_{0}\right)$, first quartile $\left(Q_{1}\right)$, median $\left(Q_{2}\right)$, third quartile $\left(Q_{3}\right)$ and maximum $\left(Q_{4}\right)$. Also, interquartile range (IQR), the distance between upper and lower quartile $\left(Q_{3}-Q_{1}\right)$, can be seen from this plot.

Consequently, investigating the box plots presented in Figure 3 provides helpful information that can be used as an evaluation tool to assess different AI models' performance in both limited and big datasets.

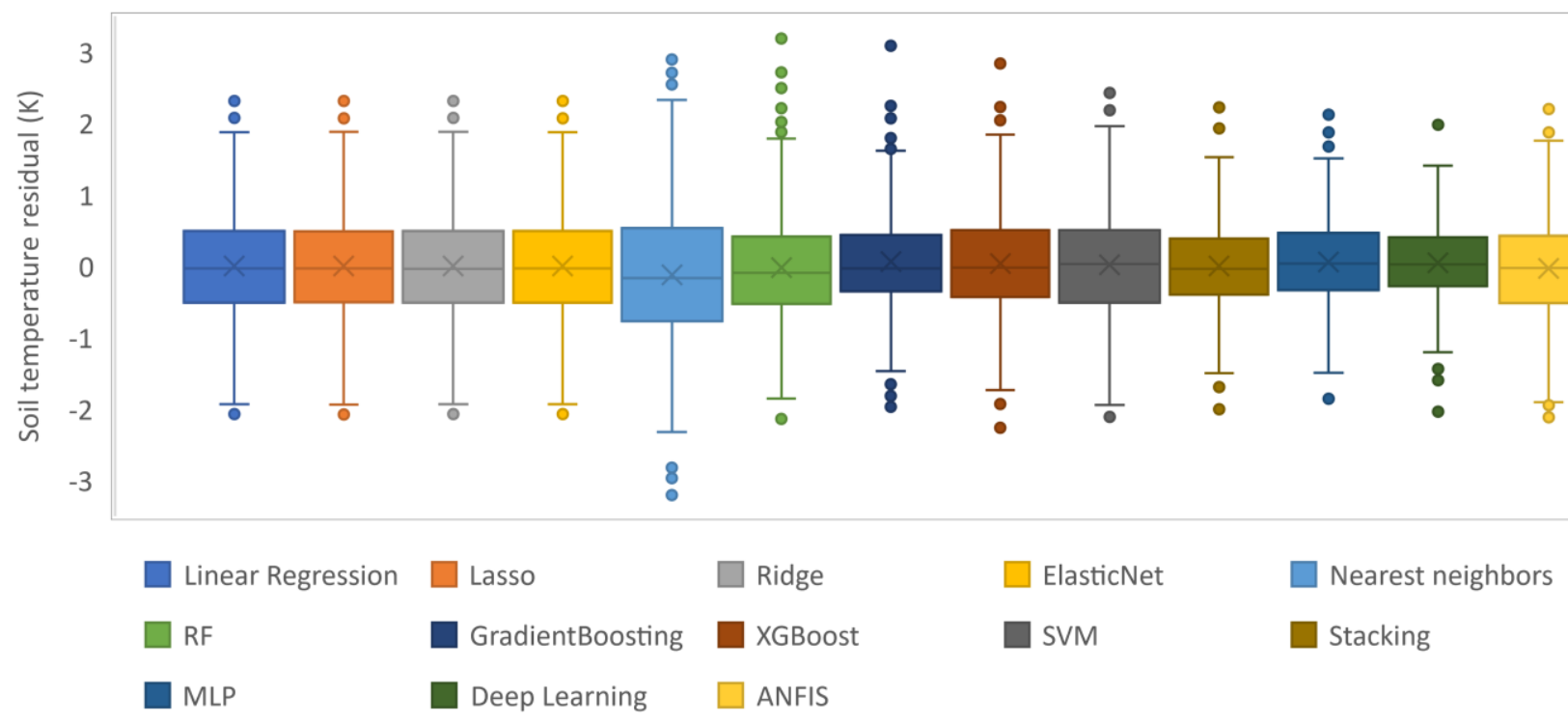


(a)

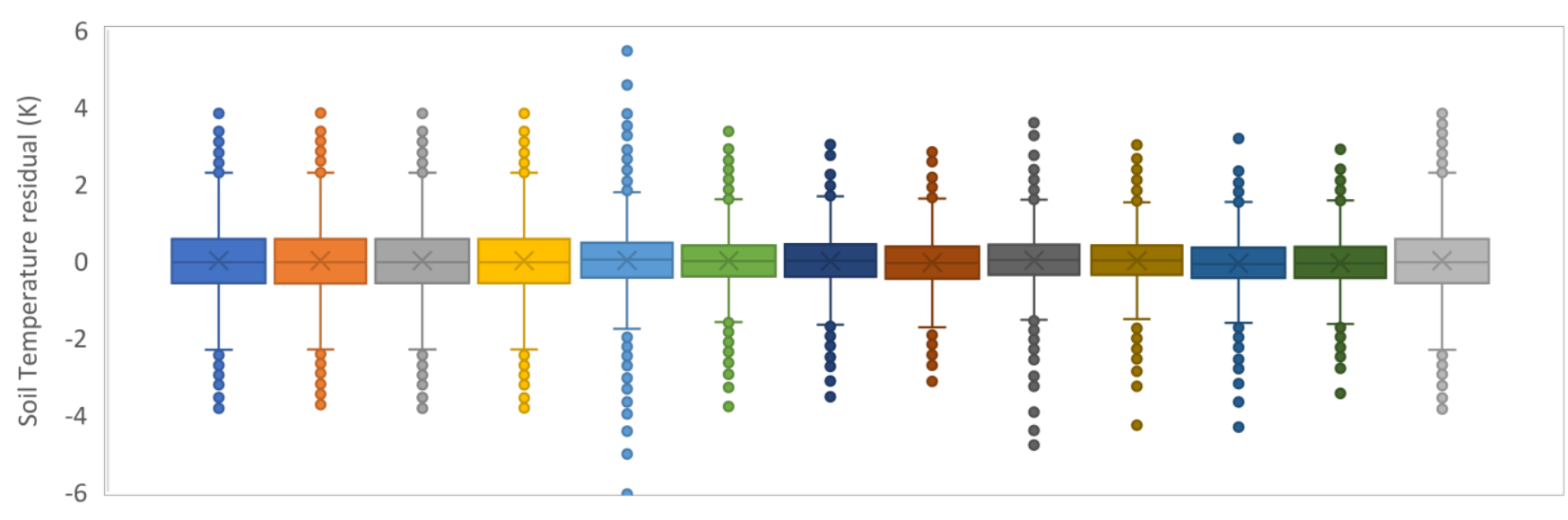

(b)

Figure 3. Box plots of soil temperature residuals computed for different AI models on a) limited dataset b) big dataset.

Figure 3-(a) demonstrates the box plot of residuals computed by different AI models on the limited dataset. It can be seen from Figure 3-(a) that deep learning residuals have the smallest interquartile range; their median is almost zero and the absolute value of their maximum and minimum are lower than other models. Therefore, this method has the best performance in predicting soil temperature. Following deep learning method, MLP and stacking methods have the least IQR, the lowest $Q_{4}$, the highest $Q_{0}$ and their $Q_{2}$ is very close to zero. So, the performance of these two methods is evaluated to be good. On the other hand, the Nearest neighbors method has the highest IQR, the highest $Q_{4}$, the least $Q_{0}$ and its $Q_{2}$ is obviously less than zero. Thus, this method did not have acceptable performance in soil temperature prediction.

Figure 3-(b) demonstrates the box plot of residuals computed by different AI models on the big dataset. It can be seen from Figure 3-(b) that the median of all models is very close to zero. The interquartile range of deep learning, MLP and stacking methods are less than others. Deep learning has the least maximum and the highest minimum among these three. Therefore, deep learning followed by MLP and stacking methods showed the best performance for big dataset as well.

Figure 4 is a scatter plot of the predicted soil temperatures computed by applying all different AI methods on ERA5 data, which demonstrates a good fit between the observed values and the models' predictions. The predicted soil temperatures show a very close match to the identity line in Figures 4(a) and 4(b). It was determined that all AI models were able to provide reliable soil temperature results on both limited dataset and big dataset.

Information presented in Figure 4 shows that the size of data in AI models plays a significant role in the correctness of results, and more data will lead to more robust and promising results. It can be seen that the correlation between actual and predicted data was $97 \%$ for the big dataset, whereas the R-squared value for the limited dataset was found to be $94.5 \%$. 


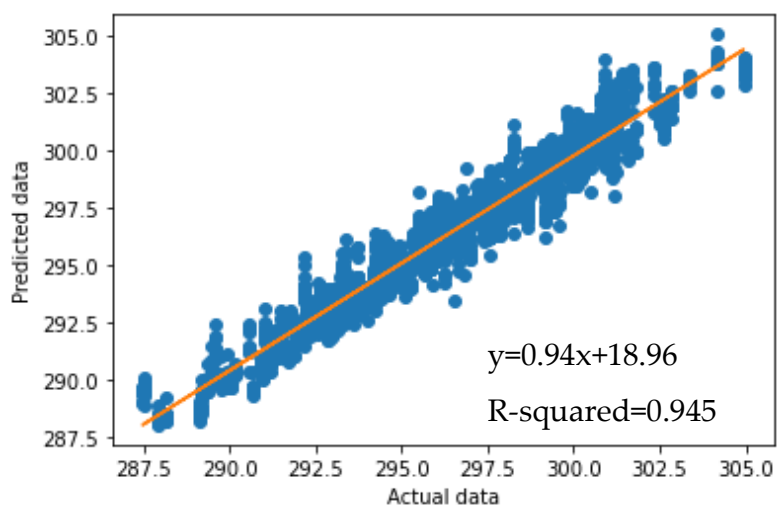

(a)

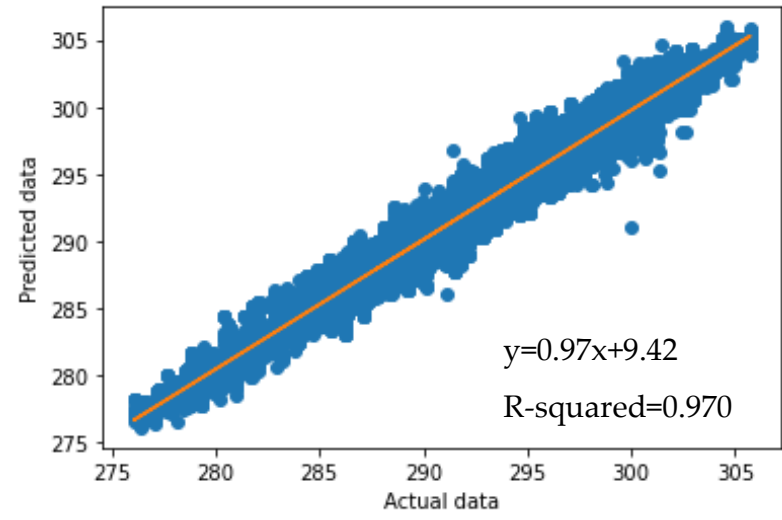

(b)

Figure 4. Scatter plots of predicted and observed soil temperature using different AI models a) limited dataset b) big dataset.

\section{Discussion}

In the current study, thirteen AI models, namely Linear regression, Lasso, Ridge, Elastic Net, K Neighbors, Random Forest, Gradient Boosting, XG Boost, Support Vector Machine, Stacking, Multi-Layer Perceptron, Deep Learning and Adaptive Neuro-Fuzzy Inference System, were employed to predict soil temperature. The mentioned models are applied to two sets of data with different quantities of information to assess the performance of the various AI models. Meanwhile, the effect of dataset size on the behavior of AI models was evaluated.

To measure the quality of different AI models, the statistical indicators of Equations 9 to 14 were applied and the results of error analysis are presented in Tables 3 for both limited and big datasets.

As seen in Table 3, R-squared values are very near 1.00 for both limited and big datasets which shows a strong correlation between the results predicted by different AI models and soil temperature data. The average R-squared for limited and big datasets calculated equals 0.94 and 0.97 , respectively. This issue is approved by scatter plots illustrated in Figure 4 and offers an overall acceptable performance for all AI methods.

Meanwhile, Table 3 indicated that while employing one AI model using two sets of input data, the AI model works better while increasing the quantity of information, leading to a more robust match between predicted results and soil temperatures. This conclusion is valid for all applied AI models, as shown in Table 3.

An examination of the error values presented in Table 3 shows that the average NRMSE for the limited dataset was $4.5 \%$, while this value equals $2.7 \%$ for the big dataset. This proves that using more data improves error measures extensively, and regardless of which AI method is applied, employing more data leads to better results.

Table 3. Error analysis of predicted soil temperature values using different AI models.

\begin{tabular}{|c|c|c|c|c|c|c|c|}
\hline $\begin{array}{c}\text { Size of } \\
\text { database }\end{array}$ & AI Models & $\begin{array}{l}\text { MaxE } \\
\text { (K) }\end{array}$ & $\begin{array}{c}\text { MAE } \\
\text { (K) }\end{array}$ & $\begin{array}{c}\text { MSE } \\
\left(\mathrm{K}^{2}\right)\end{array}$ & $\begin{array}{c}\text { RMSE } \\
\text { (K) }\end{array}$ & $\begin{array}{c}\text { NRMSE } \\
(-)\end{array}$ & $\begin{array}{l}R^{2} \\
(-)\end{array}$ \\
\hline \multirow{9}{*}{ 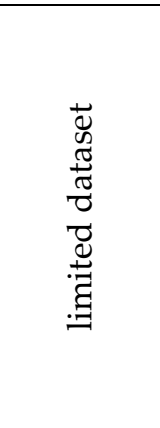 } & Linear Regression & 2.31 & 0.63 & 0.65 & 0.81 & $4.6 \%$ & 0.94 \\
\hline & Lasso & 2.31 & 0.63 & 0.65 & 0.81 & $4.6 \%$ & 0.94 \\
\hline & Ridge & 2.31 & 0.63 & 0.65 & 0.81 & $4.6 \%$ & 0.94 \\
\hline & Elastic Net & 2.31 & 0.63 & 0.65 & 0.81 & $4.6 \%$ & 0.94 \\
\hline & Nearest Neighbors & 3.21 & 0.81 & 1.09 & 1.04 & $6.0 \%$ & 0.91 \\
\hline & $\mathrm{RF}$ & 3.23 & 0.65 & 0.75 & 0.86 & $4.9 \%$ & 0.94 \\
\hline & Gradient Boosting & 3.08 & 0.58 & 0.62 & 0.79 & $4.5 \%$ & 0.95 \\
\hline & XG Boost & 2.84 & 0.61 & 0.66 & 0.81 & $4.7 \%$ & 0.94 \\
\hline & SVM & 2.42 & 0.64 & 0.65 & 0.81 & $4.6 \%$ & 0.94 \\
\hline
\end{tabular}




\begin{tabular}{|c|c|c|c|c|c|c|c|}
\hline & Stacking & 2.22 & 0.51 & 0.45 & 0.67 & $3.9 \%$ & 0.96 \\
\hline & MLP & 2.12 & 0.5 & 0.44 & 0.67 & $3.8 \%$ & 0.96 \\
\hline & Deep Learning & 2.04 & 0.46 & 0.39 & 0.62 & $3.6 \%$ & 0.97 \\
\hline & ANFIS & 2.2 & 0.62 & 0.65 & 0.81 & $4.6 \%$ & 0.94 \\
\hline \multirow{13}{*}{  } & Linear Regression & 3.89 & 0.74 & 0.95 & 0.97 & $3.3 \%$ & 0.96 \\
\hline & Lasso & 3.90 & 0.74 & 0.95 & 0.98 & $3.3 \%$ & 0.96 \\
\hline & Ridge & 3.89 & 0.74 & 0.95 & 0.97 & $3.3 \%$ & 0.96 \\
\hline & Elastic Net & 3.89 & 0.74 & 0.95 & 0.97 & $3.3 \%$ & 0.96 \\
\hline & Nearest Neighbors & 6.06 & 0.60 & 0.69 & 0.83 & $2.8 \%$ & 0.97 \\
\hline & $\mathrm{RF}$ & 3.79 & 0.53 & 0.50 & 0.71 & $2.4 \%$ & 0.98 \\
\hline & Gradient Boosting & 3.53 & 0.53 & 0.48 & 0.69 & $2.3 \%$ & 0.98 \\
\hline & XG Boost & 3.14 & 0.52 & 0.46 & 0.68 & $2.3 \%$ & 0.98 \\
\hline & SVM & 4.79 & 0.53 & 0.51 & 0.71 & $2.4 \%$ & 0.98 \\
\hline & Stacking & 4.28 & 0.49 & 0.43 & 0.66 & $2.2 \%$ & 0.98 \\
\hline & MLP & 4.32 & 0.51 & 0.45 & 0.67 & $2.3 \%$ & 0.98 \\
\hline & Deep Learning & 3.44 & 0.51 & 0.44 & 0.66 & $2.2 \%$ & 0.98 \\
\hline & ANFIS & 8.99 & 0.74 & 0.97 & 0.98 & $3.3 \%$ & 0.96 \\
\hline
\end{tabular}

Moreover, it was found from Table 3 that four models of Linear Regression, Lasso, Ridge and Elastic Net, which have linear base, showed the same error values for all evaluation metrics in both datasets, demonstrating they had very similar performance.

As mentioned previously in the methodology section, the last three methods have linear bases and are refined versions of classic linear regression due to adding regularization terms. Relatively poor MAE, MSE and NRMSE obtained by linear regression results demonstrated that this method cannot precisely predict soil temperature. At the same time, the same values of MAE, MSE and NRMSE obtained by Lasso, Ridge and Elastic Net models showed that linear regression modifications still were not appropriate tools to predict soil temperatures.

The Neighbors method had the lowest performance among the investigated AI models for the limited database with the greatest MAE, RMSE and NRMSE. Although this method did not show results with the highest error for the big database, it was one of the least performant methods among all considered AI models with the greatest Maximum error and high RMSE. This finding goes back to the logic behind the Nearest Neighbors method, which does not work in the present study. In this method, a number of nearest neighbors are involved in the model, which does not apply for this prediction since it was more temporal rather than spatial.

A closer look at error values presented in Table 3 shows that three AI methods: stacking, MLP and Deep Learning had better performance than other models. On the limited dataset, the average MAE for these three AI models is less than $0.5 \mathrm{~K}$, while other AI models had MAE of more than $0.65 \mathrm{~K}$. The situation is the same for the big database. The average MAE for these three AI models is approximately $0.5 \mathrm{~K}$, while other AI models had MAE values of approximately $0.65 \mathrm{~K}$. The other error indicator, RMSE, showed a similar trend. On both datasets, the average RMSE for these three AI models was approximately $0.65 \mathrm{~K}$, while other AI models had a RMSE of approximately $0.85 \mathrm{~K}$.

Although deep learning was the best model, the stacking method, which is an ensemble of some not very advanced models, showed good performance and predicted the soil temperature with acceptable precision. This performance was better on the big dataset.

It is worth mentioning that the computation cost should be noted as an essential parameter in picking the best method. The execution time for the limited dataset was negligible, but it was significant for the big pieces of information. The average computation time for deep learning model was $17.5 \mathrm{~s}$, while these values were $10.5 \mathrm{~s}$ and $20.5 \mathrm{~s}$ for MLP and stacking models, respectively. The stacking model suffers from insufficient execution speed despite showing adequate error metrics. 
In statistical analysis, two concepts of confidence region and prediction bands are often used. The confidence band represents the uncertainty in an estimate of the regression on the data. The prediction band is the region that contains approximately $95 \%$ of the points. If another pair of actual value -calculated value is taken, there is a $95 \%$ chance it falls within the prediction band.

The 95\% confidence region and 95\% prediction band for these three models: stacking, MLP and Deep Learning, on both limited and big datasets are depicted in Figure 5. The confidence bands in Figure 5 supports the previously mentioned claim regarding the strength of these AI models.

A sensitivity analysis has been done to find the importance level and relevance rate of atmospheric parameters used as AI models' input. In this regard, the three selected AI models of Stacking, MLP and deep learning, which had the better performance, are executed several times. For each run, one of the input parameters is omitted. Then, the code was implemented with remained 7 variables out of 8 preliminary inputs of air temperature, precipitation, surface pressure, evaporation, wind, dewpoint temperature, solar radiation and thermal radiation. The error indicators of AI model results with remained 7 input parameters are presented in Table 4.
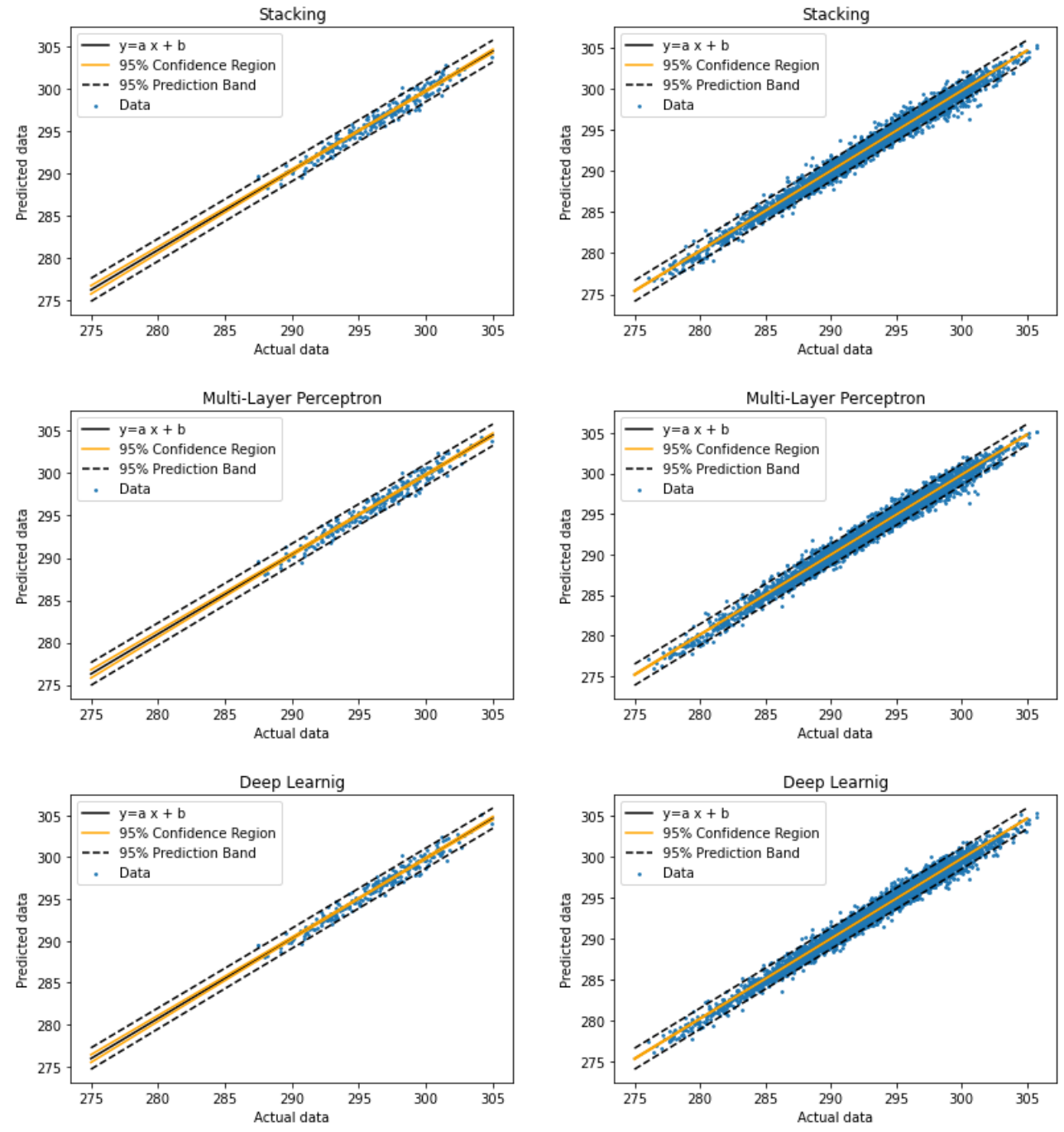

(a)

(b)

Figure 5. Confidence regions and prediction bands of different AI models a) limited dataset b) big dataset.

Table 4 shows that omitting air temperature from input variables of AI model leads to a low correlation coefficient and high NRMSE. It can be concluded that air temperature is a very relevant variable and has high importance in soil temperature prediction. The 
same trend has happened for all three AI models and both limited and big datasets applied in the sensitivity analysis.

Table 4. Sensitivity analysis of input variables for AI models.

\begin{tabular}{|c|c|c|c|c|c|c|c|c|}
\hline AI model & omitted variable & MaxE & MAE & MSE & RMSE & NRMSE & $\mathbf{R}^{2}$ & Importance \\
\hline \multirow{8}{*}{ 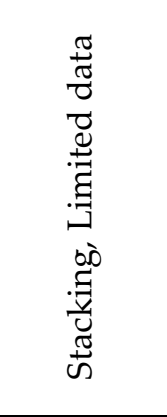 } & precipitation & 2.22 & 0.50 & 0.44 & 0.66 & $3.8 \%$ & 0.96 & \multirow[t]{5}{*}{8} \\
\hline & pressure & 2.39 & 0.56 & 0.51 & 0.72 & $4.1 \%$ & 0.96 & \\
\hline & evaporation & 2.29 & 0.56 & 0.56 & 0.75 & $4.3 \%$ & 0.95 & \\
\hline & wind & 2.16 & 0.53 & 0.48 & 0.69 & $3.9 \%$ & 0.96 & \\
\hline & dewpoint & 2.22 & 0.52 & 0.48 & 0.69 & $4.0 \%$ & 0.96 & \\
\hline & solar radiation & 2.43 & 0.60 & 0.61 & 0.78 & $4.5 \%$ & 0.95 & \multirow[t]{2}{*}{2} \\
\hline & thermal radiation & 2.12 & 0.52 & 0.46 & 0.68 & $3.9 \%$ & 0.96 & \\
\hline & air temperature & 7.31 & 1.39 & 3.24 & 1.80 & $10.3 \%$ & 0.72 & 1 \\
\hline AI model & omitted variable & MaxE & MAE & MSE & RMSE & NRMSE & $\mathbf{R}^{2}$ & Importance \\
\hline \multirow{8}{*}{ 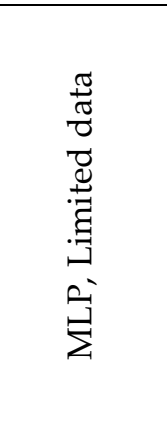 } & precipitation & 1.98 & 0.49 & 0.42 & 0.65 & $3.7 \%$ & 0.96 & \multirow[t]{5}{*}{8} \\
\hline & pressure & 2.17 & 0.53 & 0.46 & 0.68 & $3.9 \%$ & 0.96 & \\
\hline & evaporation & 2.32 & 0.54 & 0.52 & 0.72 & $4.1 \%$ & 0.95 & \\
\hline & wind & 2.12 & 0.53 & 0.47 & 0.69 & $3.9 \%$ & 0.96 & \\
\hline & dewpoint & 1.93 & 0.50 & 0.45 & 0.67 & $3.8 \%$ & 0.96 & \\
\hline & solar radiation & 2.21 & 0.58 & 0.59 & 0.77 & $4.4 \%$ & 0.95 & 2 \\
\hline & thermal radiation & 2.19 & 0.52 & 0.46 & 0.68 & $3.9 \%$ & 0.96 & \\
\hline & air temperature & 5.92 & 1.20 & 2.50 & 1.58 & $9.0 \%$ & 0.79 & 1 \\
\hline \multirow{8}{*}{ 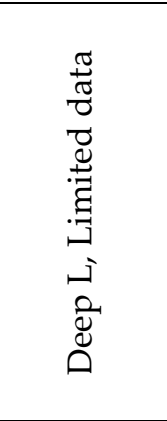 } & precipitation & 2.09 & 0.50 & 0.43 & 0.66 & $3.8 \%$ & 0.96 & \multirow[t]{5}{*}{7} \\
\hline & pressure & 2.18 & 0.52 & 0.45 & 0.67 & $3.9 \%$ & 0.96 & \\
\hline & evaporation & 2.16 & 0.52 & 0.49 & 0.70 & $4.0 \%$ & 0.96 & \\
\hline & wind & 2.01 & 0.53 & 0.50 & 0.70 & $4.0 \%$ & 0.96 & \\
\hline & dewpoint & 2.27 & 0.49 & 0.45 & 0.67 & $3.9 \%$ & 0.96 & \\
\hline & solar radiation & 2.51 & 0.59 & 0.61 & 0.78 & $4.5 \%$ & 0.95 & 2 \\
\hline & thermal radiation & 2.29 & 0.49 & 0.42 & 0.65 & $3.7 \%$ & 0.96 & 8 \\
\hline & air temperature & 5.69 & 1.33 & 2.93 & 1.71 & $9.8 \%$ & 0.75 & 1 \\
\hline \multirow{8}{*}{ 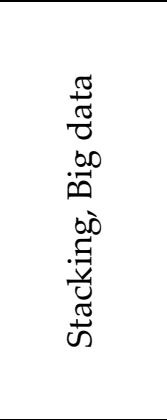 } & precipitation & 4.16 & 0.49 & 0.43 & 0.66 & $2.2 \%$ & 0.98 & \multirow{7}{*}{2} \\
\hline & pressure & 4.21 & 0.52 & 0.47 & 0.68 & $2.3 \%$ & 0.98 & \\
\hline & evaporation & 3.76 & 0.54 & 0.54 & 0.73 & $2.5 \%$ & 0.98 & \\
\hline & wind & 3.80 & 0.54 & 0.53 & 0.73 & $2.4 \%$ & 0.98 & \\
\hline & dewpoint & 3.99 & 0.53 & 0.51 & 0.71 & $2.4 \%$ & 0.98 & \\
\hline & solar radiation & 4.23 & 0.59 & 0.64 & 0.80 & $2.7 \%$ & 0.97 & \\
\hline & thermal radiation & 4.26 & 0.52 & 0.49 & 0.70 & $2.4 \%$ & 0.98 & \\
\hline & air temperature & 10.24 & 1.19 & 2.78 & 1.67 & $5.6 \%$ & 0.88 & 1 \\
\hline \multirow{8}{*}{ 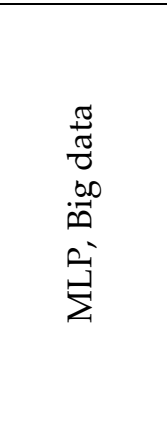 } & precipitation & 3.82 & 0.53 & 0.49 & 0.70 & $2.3 \%$ & 0.98 & \multirow[t]{5}{*}{8} \\
\hline & pressure & 4.27 & 0.53 & 0.49 & 0.70 & $2.4 \%$ & 0.98 & \\
\hline & evaporation & 4.10 & 0.60 & 0.64 & 0.80 & $2.7 \%$ & 0.97 & \\
\hline & wind & 3.88 & 0.58 & 0.59 & 0.77 & $2.6 \%$ & 0.97 & \\
\hline & dewpoint & 3.82 & 0.59 & 0.62 & 0.79 & $2.6 \%$ & 0.97 & \\
\hline & solar radiation & 4.34 & 0.65 & 0.74 & 0.86 & $2.9 \%$ & 0.97 & \multirow[t]{2}{*}{2} \\
\hline & thermal radiation & 4.13 & 0.56 & 0.55 & 0.74 & $2.5 \%$ & 0.98 & \\
\hline & air temperature & 10.50 & 1.32 & 3.19 & 1.78 & $6.0 \%$ & 0.86 & 1 \\
\hline
\end{tabular}




\begin{tabular}{|c|c|c|c|c|c|c|c|c|}
\hline \multirow{8}{*}{ 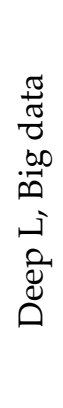 } & precipitation & 3.94 & 0.52 & 0.46 & 0.68 & $2.3 \%$ & 0.98 & 8 \\
\hline & pressure & 4.33 & 0.52 & 0.46 & 0.68 & $2.3 \%$ & 0.98 & \\
\hline & evaporation & 3.74 & 0.57 & 0.58 & 0.76 & $2.6 \%$ & 0.97 & \\
\hline & wind & 3.71 & 0.58 & 0.58 & 0.76 & $2.6 \%$ & 0.97 & \\
\hline & dewpoint & 3.58 & 0.57 & 0.54 & 0.73 & $2.5 \%$ & 0.98 & \\
\hline & solar radiation & 4.43 & 0.61 & 0.65 & 0.81 & $2.7 \%$ & 0.97 & 2 \\
\hline & thermal radiation & 3.84 & 0.52 & 0.48 & 0.69 & $2.3 \%$ & 0.98 & \\
\hline & air temperature & 10.03 & 1.28 & 2.96 & 1.72 & $5.8 \%$ & 0.87 & 1 \\
\hline
\end{tabular}

Comparing the obtained errors with the original error values, considering all 8 input parameters presented in Table 3, shows that following air temperature, solar radiation is the most critical variable in soil temperature prediction.

Also, sensitivity analysis displays that precipitation has a negligible effect on results. So, the precipitation does not play an important role in soil temperature forecast and can be omitted from the prediction models without decreasing precision. The mentioned importance level is the same for all three AI models and in both datasets. The level of importance and relevance of each variable is introduced in the last column.

\section{Conclusions}

A precise and cost-effective model for soil temperature forecasting, which enjoys the benefits of artificial intelligence techniques, is developed in the present research. Therefore, 13 AI models including Linear regression, Ridge, Lasso, Elastic Net, Nearest Neighbors, Random Forest, Gradient Boosting, XG Boosting, stacking method, SVM, MLP, Deep Learning and ANFIS are employed to generate a comprehensive and detailed assessment of the performance of different AI approaches in soil temperature estimation. In this regard, 8 hourly land and atmospheric variables of air temperature, precipitation, surface pressure, evaporation, wind gust, dewpoint temperature, solar radiation and thermal radiation were employed, and predictions were made using two limited and big datasets. The results showed that $\mathrm{AI}$ is a promising approach in climate parameter forecast and developed AI models showed a reliable ability in soil temperature prediction. Also, applying AI models to more information led to better results even using the same method.

The key findings of this study are summarized as follows:

- Among all 13 AI models applied in the current study, deep learning followed by the MLP method, showed the best performance in predicting soil temperature with the highest correlation coefficient and lowest error metrics.

- Although deep learning was the best model, stacking method showed good performance with an acceptable precision in soil temperature prediction.

- Sensitivity analysis shows air temperature and solar radiation plays the most important roles in soil temperature prediction, while precipitation can be neglected in forecast AI models.

The present research has studied the entire data in the considered time history. Future work will focus on the extreme data and investigate the developed AI models' performance in the first and last quarters.

Author Contributions: Conceptualization, J.C., A.M., H.S. and P.P.; methodology, H.I.; software, H.I.; validation, H.I. and P.P.; formal analysis, H.I. and P.P.; investigation, H.I.; resources, H.I.; data curation, H.I.; writing - original draft preparation, H.I. and A.M.; writing - review and editing J.C., P.P., H.S. and A.M.; visualization, H.I.; supervision, A.M. and J.C.; project administration, A.M., J.C. and H.S.; funding acquisition, A.M., H.S. and J.C. All authors have read and agreed to the published version of the manuscript.

Funding: This research was funded by National Research Council Canada through the Artificial Intelligence for Logistics Supercluster Support Program, grant number AI4L-120.

Conflicts of Interest: The authors declare no conflict of interest. 


\section{References}

1. Hao H, Yu F, Li Q. Soil Temperature Prediction Using Convolutional Neural Network Based on Ensemble Empirical Mode Decomposition. IEEE Access. 2021;9:4084-4096. doi:10.1109/ACCESS.2020.3048028

2. Yu F, Hao H, Li Q. An Ensemble 3D Convolutional Neural Network for Spatiotemporal Soil Temperature Forecasting. Sustainability. 2021;13(16):9174. doi:10.3390/su13169174

3. Alizamir M, Kisi O, Ahmed AN, Mert C, Fai CM, Kim S, Kim NW, El-Shafie A. Advanced machine learning model for better prediction accuracy of soil temperature at different depths. PLOS ONE. 2020;15(4). doi:10.1371/journal.pone.0231055

4. Seifi A, Ehteram M, Nayebloei F, Soroush F, Gharabaghi B, TorabiHaghighi A. GLUE uncertainty analysis of hybrid models for predicting hourly soil temperature and application wavelet coherence analysis for correlation with meteorological variables. Soft Computing. 2021;25(16):10723-10748. doi:10.1007/s00500-021-06009-4

5. Abimbola O, Meyer G, Mittelstet A, Rudnick D, Franz T. Knowledge-guided machine learning for improving daily soil temperature prediction across the United States. Vadose Zone Journal. 2021;20(5). doi: 10.1002/vzj2.20151

6. Zeynoddin M, Ebtehaj I, Bonakdari H. Development of a linear based stochastic model for daily soil temperature prediction: One step forward to sustainable agriculture. Computers and Electronics in Agriculture. 2020;176. doi:10.1016/j.compag.2020.105636

7. Feng Y, Cui N, Hao W, Gao L, Gong D. Estimation of soil temperature from meteorological data using different machine learning models. Geoderma. 2019;338: 67-77. doi:10.1016/j.geoderma.2018.11.044

8. Mehdizadeh S, Fathian F, Safari M, Khosravi A. Developing novel hybrid models for estimation of daily soil temperature at various depths. Soil E Tillage Research. 2020;197. doi: 10.1016/j.still.2019.104513

9. Bonakdari H, Moeeni H, Ebtehaj I, Zeynoddin M, Mahoammadian A, Gharabaghi B. New insights into soil temperature time series modeling: linear or nonlinear? Theoretical and Applied Climatology. 2019;135(3-4):1157-1177. doi:10.1007/s00704-018-2436-2

10. George RK. Prediction of Soil Temperature by using Artificial Neural Network Algorithms. Nonlinear Analysis. 2001;47:17371748.

11. Bilgili M. Prediction of soil temperature using regression and artificial neural network models. Meteorology and Atmospheric Physics. 2010;110(1):59-70. doi:10.1007/s00703-010-0104-x

12. Ozturk M, Salman O, Koc M. Artificial neural network model for estimating the soil temperature. Canadian Journal of Soil Science. 2011;91(4):551-562. doi:10.4141/cjss10073

13. ZareAbyaneh H, BayatVarkeshi M, Golmohammadi G, Mohammadi K. Soil temperature estimation using an artificial neural network and co-active neuro-fuzzy inference system in two different climates. Arabian Journal of Geosciences. 2016;9(5). doi:10.1007/s12517-016-2388-8

14. Citakoglu H. Comparison of artificial intelligence techniques for prediction of soil temperatures in Turkey. Theoretical and Applied Climatology. 2017;130(1-2):545-556. doi:10.1007/s00704-016-1914-7

15. Himika, Kaur S, Randhawa S. Global Land Temperature Prediction by Machine Learning Combo Approach. In: 9th International Conference on Computing, Communication and Networking Technologies, Bengaluru, July 10-12, IEEE, 2018:1-8.

16. Delbari M, Sharifazari S, Mohammadi E. Modeling daily soil temperature over diverse climate conditions in Iran - a comparison of multiple linear regression and support vector regression techniques. Theoretical and Applied Climatology. 2019;135(3-4):9911001. doi:10.1007/s00704-018-2370-3

17. Li C, Zhang Y, Ren X. Modeling hourly soil temperature using deep BiLSTM neural network. Algorithms. 2020;13(7). doi:10.3390/A13070173

18. Penghui L, Ewees AA, Beyaztas BH, Qi C, Salih SQ, Al-Ansari N, Bhagat SK, Yaseen ZM, Singh VP. Metaheuristic optimization algorithms hybridized with artificial intelligence model for soil temperature prediction: Novel model. IEEE Access. 2020;8:5188451904. doi:10.1109/ACCESS.2020.2979822

19. Shamshirband S, Esmaeilbeiki F, Zarehaghi D, Neyshabouri M, Samadianfard S, Ghorbani MA, Mosavi A, Nabipour N, Chau KW. Comparative analysis of hybrid models of firefly optimization algorithm with support vector machines and multilayer perceptron for predicting soil temperature at different depths. Engineering Applications of Computational Fluid Mechanics. 2020;14(1):939-953. doi:10.1080/19942060.2020.1788644

20. Google Maps. Available online: https://www.google.ca/maps/@45.3759264,-75.7182361,11.33z (Accessed on 1-Jul-2021)

21. Hersbach H, Bell B, Berrisford P, Biavati G, Horányi A, Muñoz Sabater J, Nicolas J, Peubey C, Radu R, Rozum I, Schepers D, Simmons A, Soci C, Dee D, Thépaut J-N. ERA5 hourly data on single levels from 1979 to present. Copernicus Climate Change Service (C3S) Climate Data Store (CDS). 2018; doi:10.24381/cds.adbb2d47 (Accessed on 1-Jul-2021).

22. Tabari H, Sabziparvar A, Ahmadi M. Comparison of artificial neural network and multivariate linear regression methods for estimation of daily soil temperature in an arid region. Meteorology and Atmospheric Physics. 2011;110:135-142. doi:10.1007/s00703010-0110-z

23. Quan Q, Hao Z, Xifeng H, Jingchun L. Research on water temperature prediction based on improved support vector regression. Neural ComputEApplic. 2020; Special Issue: New trends in brain computer interface. doi:10.1007/s00521-020-04836-4

24. Radhika Y, Shashi M. Atmospheric Temperature Prediction using Support Vector Machines. International Journal of Computer Theory and Engineering. 2009;1(1): 5-58. doi:10.7763/IJCTE.2009.V1.9

25. Cho D, Yoo C, Im J, Lee Y, Lee J. Improvement of spatial interpolation accuracy of daily maximum air temperature in urban areas using a stacking ensemble technique. Giscience E Remote Sensing. 2020;57(5):633-649. doi:10.1080/15481603.2020.1766768

26. Bayatvarkeshi M, Bhagat S, Mohammadi K, Kisi O, Farahani M, Hasani A, Deo R, Yaseen Z. Modeling soil temperature using air temperature features in diverse climatic conditions with complementary machine learning models. Computers and Electronics in Agriculture. 2021;185. doi: 10.1016/j.compag.2021.106158 
27. Wang X, Li W, Li Q. A New Embedded Estimation Model for Soil Temperature Prediction. Scientific Programming. 2021. doi:10.1155/2021/5881018

28. Samadianfard S, Ghorbani M, Mohammadi B. Forecasting soil temperature at multiple-depth with a hybrid artificial neural network model coupled hybrid firefly optimizer algorithm. Information Processing in Agriculture. 2018;5: 465-476. doi: 10.1016/j.inpa.2018.06.005

29. Ikechukwu M, Ebinne E, Yun Z, Patrick B. Prediction of Land Surface Temperature (LST) Changes within Ikom City in Nigeria Using Artificial Neural Network (ANN). International Journal of Remote Sensing Applications. 2016;6. doi:10.14355/ijrsa.2016.06.010

30. Sada S, Ikpeseni S. Evaluation of ANN and ANFIS modeling ability in the prediction of AISI 1050 steel machining performance. Heliyon. 2021;7(2). doi:10.1016/j.heliyon.2021.e06136

31. Mustafa E, Co Y, Liu G, Kaloop M, Beshr A, Zarzoura F, Sadek M. Study for Predicting Land Surface Temperature (LST) Using Landsat Data: A Comparison of Four Algorithms. Advances in Civil Engineering. 2020. doi:10.1155/2020/7363546

32. Gholami A, Bonakdari H, Ebtehaj I, Mohammadian M, Gharabaghi B, Khodashenas S. Uncertainty analysis of intelligent model of hybrid genetic algorithm and particle swarm optimization with ANFIS to predict threshold bank profile shape based on digital laser approach sensing. Measurement. 2018;121: 294-303. doi:10.1016/j.measurement.2018.02.070 